\title{
El impacto de Twitter en el periodismo: un estado de la cuestión
}

The impact of Twitter on Journalism: A State of Play

Amparo López Meri, Universitat Jaume I - meri@uji.es

Abstract

The academic literature about the implications of Twitter in journalism has proliferated in recent years. The aim of this paper is to explain the main trends and researches in this field. Most of the studies analyses thoroughly how journalists and media use this network. The findings indicate the use of Twitter by journalists in newsroom to find information and sources, breaking news, disseminate and promote contents becomes normal. On the other hand, they point out Twitter is changing journalism classical rules and values, because it allows citizens to participate in the news construction. In this sense, researches on the coverage of citizen protests and natural disasters show that Twitter contributes to select, focus and verify information collaboratively and collectively. From this perspective, news would be the result of a real time public negotiation between journalists and users who act as sources. The future lies in conducting further investigations about these trends and new journalists roles.

Keywords

Twitter, journalism, journalism research, sourcing, gatekeeping.

Resumen

La literatura académica sobre las implicaciones de Twitter en el periodismo ha proliferado en los últimos años. El objetivo de este artículo es exponer las principales tendencias y líneas de investigación en este ámbito. La mayor parte de los estudios ahonda en los usos que periodistas y medios asignan a esta red social. Al respecto, los resultados indican que Twitter se normaliza en las redacciones, para localizar datos y fuentes, informar, distribuir y promocionar contenidos. Por otro lado, se apunta que Twitter está modificando las normas y valores clásicos del periodismo, porque permite la participación de los ciudadanos en la construcción de las noticias. En este sentido, investigaciones sobre la cobertura de protestas ciudadanas y desastres naturales muestran que Twitter posibilita la selección de noticias, el enfoque, la obtención de información y su verificación de manera colaborativa y colectiva. Desde esta perspectiva, las noticias serían fruto de la negociación pública y en tiempo real entre periodistas y usuarios que ejercen de fuentes. El futuro pasa por seguir investigando estas tendencias y los nuevos roles del periodista.

Palabras clave

Twitter, periodismo, investigación periodística, sourcing, gatekeeping.

Sumario

1. Introducción. 2. La influencia de Twitter en las rutinas periodísticas. 3. La naturaleza de Twitter en el punto de mira. 4. Conclusiones: limitaciones y tendencias futuras en la investigación sobre Twitter y periodismo. 5. Bibliografía. 


\section{Introducción}

Las implicaciones de Twitter en el periodismo son palpables en todos los estadios del proceso comunicativo, tanto en la producción, como en la difusión y el consumo de contenidos informativos. Estos tres ámbitos han captado el interés de la investigación en Ciencias de la Comunicación, aunque el primero es el que ha generado más literatura, debido a las transformaciones que el microblogging está imponiendo en el proceso de construcción de noticias y en las rutinas de periodistas y grupos mediáticos.

Twitter cuenta con 270 millones de usuarios activos en 2014, que intercambian más de 400 millones de mensajes diarios (Wickre, 2013). Pese a su expansión, su implantación no es generalizada y se circunscribe a un grupo selecto de población, de entre 35 y 50 años. En contrapartida, el usuario medio de Twitter es de dos a tres veces más propenso a visitar sitios web de noticias que otro ciudadano medio (Farhi, 2009). De ahí, el interés de los medios por promocionar sus contenidos y crearse una marca en Twitter.

Con el objetivo de actualizar y ordenar las principales conclusiones de profesionales y académicos, este artículo revisa la literatura más relevante en la materia, indica los temas todavía pendientes y los aspectos por explorar en relación al impacto de Twitter en el periodismo. Respecto a la literatura publicada, puede estructurarse en dos grandes ámbitos de estudio: las rutinas periodísticas y la naturaleza del periodismo. El primero se centra en cómo utilizan Twitter los periodistas y medios de comunicación (Bruns, 2005; Newman, 2009; Ahmad, 2010; García de Torres et al., 2011; Greer y Ferguson, 2011; Broersma y Graham, 2012; Carrera Álvarez, Sainz de Baranda, Hererro y Limón, 2012; Lasorsa, Lewis y Holton, 2012; Messner, Linke y Eford, 2012; Túñez, 2012; Artwick, 2013; Hedman y Djerf-Pierre, 2013; Noguera, 2013; Said, Serrano, García de Torres, Yerezers'ka y Calderín, 2013).

El segundo ámbito de estudio incluye las aportaciones de autores que se preguntan si está mutando la naturaleza del periodismo, si están cambiando las normas y prácticas periodísticas clásicas debido a la influencia de Twitter (Shoemaker y Vos, 2009; Sheffer y Schultz, 2010; Singer et al., 2011; Hermida, 2013; Herrera y Requejo, 2012; Lasorsa et al., 2012; Gulyas, 2013; Vis, 2013; Hermida, Lewis y Zamith, 2014). Dentro de este campo, se presta atención a la participación de los ciudadanos en la construcción del relato informativo (Domingo et al., 2008; Hermida y Thurman, 2008; Newman, 2009; Lotan et al., 2011; Brown Smith, 2012; Papacharissi y De Fatima Oliveira, 2012; Starbird, Muzny y Palen, 2012) y a la concepción de Twitter como una forma de periodismo ambiental (Burns, 2010; Hermida, 2010).

\section{La infuencia de Twitter en las rutinas periodísticas}

En el ámbito de las rutinas periodísticas se ha estudiado quién usa Twitter en las redacciones, cómo usan esta herramienta y con qué finalidades. Se ha analizado la actividad de las cuentas corporativas y el comportamiento de los redactores y periodistas especializados, así como de los editores de contenidos y directores de medios. Respecto a la actividad de los medios, las primeras investigaciones destacan el potencial de Twitter para reforzar relaciones con los consumidores y crear o consolidar su marca (Ahmad, 2010; Greer y Ferguson, 2011), y como canal de distribución de contenidos noticiosos (Messner et al., 2012).

En el ámbito internacional, se han investigado las rutinas de los principales medios de Estados Unidos (Holcomb, Gross y Mitchell, 2011; Messner et al., 2012), con análisis específicos en redacciones de televisiones locales (Greer y Ferguson, 2011), emisoras de radio (Ferguson y Greer, 2011) y comunidades de periódicos (Greer y Yan, 2010). También se ha revisado cómo aprovechan Twitter los medios latinoamericanos (García de Torres et al., 2011; Said et al., 2013) y los periódicos de referencia, como The Guardian en Reino Unido (Ahmad, 2010). Estos estudios indican que los medios recurren a Twitter con cuatro propósitos: difundir noticias, hacer marketing, interactuar con nuevos consumidores y buscar información y fuentes (Broersma y Graham, 2012).

La adhesión de los medios de comunicación a Twitter guarda relación con las necesidades de los usuarios: mantener charlas cotidianas, conversar, compartir información e informar (Java, Song, Fnin y Tseng, 2007). Frente a la noticia, los usuarios desempeñan tres funciones (Bruns y Burgess, 2012): aportan información de primera mano cuando son testigos, debaten sobre acontecimientos en curso y discuten en directo asuntos de interés periodístico, ejerciendo el gatewatching (Bruns, 2005). El término gatewatching se refiere a la incorporación de los ciudadanos-usuarios al proceso de evaluación, selección y jerarquización de contenidos, tradicionalmente monopolizado por los periodistas (gatekeeping). Las aportaciones de los usuarios conforman la banda sonora que se escucha de fondo, y cumplen la llamada función ambiente (Burns, 2010; Hermida, 2010).

Los medios todavía son conservadores respecto al uso de redes sociales. Sus cuentas corporativas funcionan como canales RSS o redistribuidores de información, para promocionarse (López y Alonso, 2013; Noguera, 2013), con enlaces de regreso a sus web, pero sin propiciar la conversación colectiva ni crear comunidades activas (Salaverría, García Avilés y Masip, 2010; García de Torres et al., 2011; Holcomb et al., 2011; Messner et al., 2012; Requejo y Herrera, 2014). Los medios comprenden el potencial de las redes sociales y las integran entre sus estrategias comerciales, pero aún están en una fase incipiente de aprovechamiento (Flores, 2009), en parte, por la falta de cultura profesional y empresarial, pues muchos periodistas siguen mediados por modelos tradicionales ajenos a escenarios digitales (Said et al., 2013). Algunos autores suscriben que se debería implementar la interacción con el público para obtener rendimiento económico (García Avilés, Kaltenbrunner y Meier, 2014).

Respecto a los estudios centrados en la figura del periodista, confirman la normalización del uso de Twitter, especialmente para informar, buscar información y encontrar fuentes (Newman, 2009; Broersma y Graham, 2012; Brown Smith, 2012; Lasorsa et al., 2012). Según Broersma y Graham (2012), los periodistas van de ronda por Twitter en busca de noticias o ideas para nuevos enfoques, igual que van de ronda al juzgado, el ayuntamiento, la comisaría de policía o el Congreso (2012: 405). Sin embargo, como explican Carrera Álvarez, Sainz de Baranda, Hererro y Limón (2012), lo inédito es poco frecuente en Twitter, pues los periodistas, más que crear nuevos contenidos, diseminan y revitalizan argumentos preexistentes, sin aprovechar la oportunidad que brinda el crowdsourcing para generar informaciones en colaboración con los usuarios.

No obstante, Twitter resulta gratificante a los periodistas, por el contacto con sus seguidores y la «confortable libertad» que les confiere 
para crear su propia marca, bajo el paraguas protector de sus medios (Carrera Álvarez et al., 2012: 51). En una encuesta a 50 periodistas españoles, Carrera Álvarez et al. (2012) comprueban que el 88\% usa Twitter para sondear el ambiente y detectar tendencias, el $86 \%$ para buscar información, el $84 \%$ para enganchar al público, el $80 \%$ para viralizar contenidos propios, el $72 \%$ para obtener ideas nuevas y el $70 \%$ para difundir información de otros medios. Sin embargo, se utiliza en menor medida para difundir exclusivas o noticias de última hora $(58 \%)$, contactar con fuentes ciudadanas (52\%), conseguir información sobre las agendas institucionales (42\%) o investigar $(24 \%)$.

Los porcentajes de la normalización se repiten en otros países. Una encuesta a periodistas de Finlandia, Alemania, Suecia y Reino Unido, indica que el $96 \%$ de los profesionales utiliza algún medio social para su trabajo (Gulyas, 2013). En Suecia, otra investigación constata que, pese a que el $85 \%$ de los periodistas recurre a Twitter habitualmente, únicamente uno de cada diez tuitea diariamente, y sólo el $2 \%$ lo hace los siete días de la semana (Hedman y Djerf-Pierre, 2013).

También se investigan las rutinas de los periodistas especializados. Los corresponsales en el extranjero difunden más noticias de última hora en Twitter que los reporteros de la redacción, y sólo incluyen puntos de vista personales en el 10\% de los tuits (Cozma y Chen, 2013). A diferencia de los corresponsales, los periodistas deportivos aportan más novedades en las ediciones impresas (Sheffer y Schultz, 2010).

Respecto al diálogo con el público, después de analizar 2.700 tuits publicados por los 51 periodistas más seguidos de Estados Unidos, Artwick (2013) encuentra evidencias de que que en Twitter, además de promocionar sus noticias y dar voz a fuentes oficiales, los periodistas se aproximan a los ciudadanos, al tuitear eventos en vivo y retuitear los testimonios y valoraciones de los usuarios. Casi un tercio de los tuits se corresponde con coberturas en directo o informaciones de última hora. Los reporteros aclaran la potencial desinformación que circula en la red, y ofrecen contexto con fotografías y otros archivos multimedia. Como advierte esta autora, no es nuevo que un periodista informe como testigo de los hechos. La novedad radica en ejercer esta función en vivo en un entorno de red, «donde los ciudadanos, funcionarios y periodistas pueden contribuir juntos a la búsqueda de la verdad» (2013: 223-225).

Otros trabajos destacan el papel activo de los usuarios como comentaristas que contribuyen a la interpretación compartida de la actualidad (Hermida et al., 2011; Hermida et al., 2014). Sin embargo, se aprecia que son minoría los periodistas que solicitan información a los usuarios (Cozma y Chen, 2013; Noguera, 2013), restando presencia a la co-creación de noticias (Hermida, 2013). Aún así, interactúan con sus seguidores más que los medios (Lasorsa et al., 2012; Artwick, 2013).

Los periodisats son más visibles desde sus cuentas personales. Con el objetivo de crearse una imagen de independencia, muchos optan por configurar su perfil sin citar a sus empresas (Rodríguez y García, 2013), incluso enlazan contenidos de otros medios si los consideran relevantes. Por eso, Noguera (2013: 110-111) concluye que los medios sociales unen a la competencia. Otros autores, en cambio, defienden que cuando los periodistas más influyentes en Twitter mencionan a la competencia es porque primero la competencia los cita a ellos, como fuentes, especialmente durante la cobertura de altercados y protestas (Vis, 2013: 38-42).

\section{La naturaleza del periodismo en el punto de mira}

Diversos estudios incorporan al debate la mutación que están sufriendo las normas y prácticas clásicas de la profesión, como la objetividad, la verificación, la búsqueda de información o sourcing y la selección y jerarquización de contenidos o gatekeeping.

\section{Opinión contra objetividad}

Respecto al principio de objetividad, apuntan que se conculca, porque los periodistas, cada vez más, tienden a opinar, posicionarse y comentar asuntos personales (Sheffer y Schultz, 2010; Lasorsa et al., 2012; Vis, 2013). Lasorsa, Lewis y Holton (2012: 30-32), que analizan el quehacer de los 500 periodistas más seguidos en Twitter, observan que los periodistas opinan libremente en el 16\% de sus tuits, aunque el $43 \%$ contiene algún elemento valorativo. En España, un trabajo de Rodríguez y García (2013: 968) suscribe la misma tendencia, tras comprobar que el $45 \%$ de los tuits difundidos por periodistas introduce comentarios personales.

Según puntualiza Hermida, los periodistas «están negociando un espacio donde la separación entre lo personal y lo privado es frágil, y donde la jerarquía entre los roles de reportero, editor y público es borrosa» (2013: 301). Tuitean sobre sus aficiones y se muestran cercanos ante su comunidad social (Lasorsa, 2012; Lasorsa et al., 2012; Cozma y Chen, 2013). Adoptando un tono más humano construyen su marca o identidad social (Ekman y Widholm, 2014). Se trata de un cambio importante, pues los periodistas, tradicionalmente, se han mostrado imparciales, en representación de sus medios. La presencia de opiniones y emociones en la cobertura informativa apunta a una simbiosis entre valores clásicos y nuevos (Papacharissi y De Fátima Oliveira, 2012). La subjetividad y la personalización se consideran formas de «periodismo ambiental» (Burns, 2010; Hermida, 2010), y reflejan los «límites cada vez más borrosos entre lo profesional y lo personal, entre periodistas, actores políticos y público» (Ekman y Widholm, 2014: 6).

Periodismo ambiental y sourcing colaborativo

La concepción de Twitter como periodismo ambiental supone darle valor de noticia a la suma de tuits (Hermida, 2010). Según esta teoría, la inteligencia colectiva surge de la conexión con otros usuarios. Por eso, la suma de tuits que se produce durante el debate de un tema de actualidad tiene más valor que la información individual y fragmentada, lo que ayuda a una mejor compensión de la realidad por parte de los periodistas. Desde esta perspectiva, Twitter alerta a los periodistas de las tendencias que subyacen a las personas y las noticias, por lo que aporta «formas más complejas de comprensión» de la comunicación pública (Hermida, 2010: 303). La conversación compartida contribuye a localizar cambios de tono o enfoque mientras se discuten asuntos de actualidad (Burns, 2010; Bruns y Burgess 2012).

En este sentido, Twitter se utiliza como fuente de noticias e información. En ciertas situaciones, los usuarios asumen el papel de sensores sociales de la noticia (Sakaki, Okazaki y Matsuo, 2010) y testigos de los hechos (Hermida et al., 2011; Singer et al., 2011), mientras la red posibilita el intercambio de datos en tiempo real, durante el desarrollo de los acontecimientos. En línea con las nociones de periodismo ambiental y crowdsourcing, los periodistas aprovechan la «sabiduría de la multitud» para completar sus historias (Broersma y Graham, 
2012: 404). En su análisis sobre el uso de \#memstorm, hashtag que alude a las tormentas de 2011 en Memphis, Brown Smith (2012: 2528) observa que los ciudadanos producen pequeñas cantidades de contenido, que en conjunto constituyen una forma de periodismo que ayuda a la comunidad a mantener la conciencia de los hechos importantes. Algunos entrevistados por Brown Smith afirmaron que confiaban en sus vecinos, tanto o más que en los periodistas.

El sourcing o aprovisionamiento de datos forma parte de la esencia de la actividad periodística, y la arquitectura de Twitter ofrece la oportunidad de investigar la relación entre periodistas y fuentes desde una perspectiva nueva, porque las interacciones entre ambos empiezan a darse en público. Los periodistas mencionan a los usuarios y establecen contacto para solicitar datos, antecedentes y contexto a la vista de la comunidad. Al respecto, frente al tradicional predominio de fuentes institucionales, Hermida, Lewis y Zamith (2014: 482) entienden que Twitter cambia el paradigma, al dejar paso a voces alternativas. Tras estudiar las interacciones de la cuenta del periodista Andy Carvin durante los levantamientos de Túnez y Egipto, estos autores observan que Carvin se aleja de las fuentes oficiales y da prioridad a los mensajes procedentes de ciudadanos, que expresan sus demandas para el cambio social y comparten sus experiencias en Twitter. En el lado opuesto, los diarios egipcios de mayor tirada decidieron confiar en fuentes convencionales (AlMaskati, 2012).

Las llamadas fuentes no-élite protagonizaron casi el $50 \%$ de los tuits y más del $70 \%$ de los retuits de Carvin, estratega de medios sociales en la National Public Radio de los Estados Unidos. Esta elevada participaron de voces alternativas en la construcción de las noticias, según Hermida et al. (2014: 493), cuestiona normas clásicas como la objetividad y la imparcialidad. Además, frente a los estudios que sostienen que Twitter se ha adaptado a las prácticas periodísticas (Newman, 2009; Singer et al., 2011; Lasorsa et al., 2012), Hermida et al. (2014) sugieren que el proceso de ensamblaje ocurre en sentido inverso, que son las prácticas periodísticas las que se están adaptando a Twitter, al menos respecto a la selección de fuentes (2014: 494). En consecuencia, tomando como base la experiencia de Carvin, podría decirse que Twitter modifica o redefine las prácticas periodísticas (Gulyas, 2013; Hermida, 2013).

Otros estudios secundan que las voces contrarias al poder tienen cabida en Twitter, donde ejercen de contrapeso frente a la versión oficial de la policía o los funcionarios. En 2010, por ejemplo, durante las protestas contra el G-20 en Toronto, los medios tradicionales insistieron en la violencia de los manifestantes, mientras que los activistas denunciaron en Twitter la violencia de la respuesta policial (Poell y Borra, 2011). También durante la llamada Arab Spring o Primavera Árabe de 2011, tanto periodistas como activistas se convirtieron en fuentes prioritarias desde sus cuentas de Twitter (Lotan et al., 2011; Papacharissi y De Fátima Oliveira, 2012).

En España, asimismo, el movimiento 15M marca un punto de inflexión y rompe el monopolio comunicativo de las fuentes oficiales, al colarse en la agenda mediática y en el debate político durante la campaña electoral de 2011, debido a su fuerza en Twitter y otras redes sociales (Casero-Ripollés y Feenstra, 2012). La monitorización que realizan los ciudadanos en los medios sociales, para vigilar, filtrar información y difundir puntos de vista alternativos, «es una forma de contrapoder que desafía a los centros de poder político y económico» (Feenstra y Casero-Ripollés, 2014: 2462).

Esta capacidad de convertir en fuentes a usuarios críticos y voces habitualmente marginadas, sugiere el potencial de Twitter para el desarrollo de un periodismo colaborativo (Castells, 2009; Hermida, 2010; Brown Smith, 2012), al menos en la cobertura de movilizaciones ciudadanas (Poell y Borra, 2011; Papacharissi y De Fátima Oliveira, 2012). En cambio, otros estudios confirman que Twitter repite los patrones de la prensa convencional, por su dependencia respecto a las fuentes oficiales. Así, Artwick (2013) detecta que el $75 \%$ de las menciones realizadas por periodistas estadounidenses recae sobre políticos y funcionarios. Este dato, sin embargo, no anula a los ciudadanos, porque los periodistas también retuitean sus mensajes. Por eso, Artwick considera que los ciudadanos «pueden estar contribuyendo al diálogo público de forma significativa» (2013: 224).

Por otro lado, se confirma que Twitter es una fuente utilizada regularmente por los periodistas. Broersma y Graham (2013), después de analizar diferentes periódicos británicos y neerlandeses entre 2007 y 2011, determinan que incluyen tuits entre sus noticias con cada vez más frecuencia. Durante este período, contabilizan hasta 5.813 tuits citados como fuentes a lo largo de 3.361 artículos. Twitter resulta una fuente atractiva para los periodistas, que siguen las discusiones sobre temas de actualidad y rastrean los tuits en busca de citas interesantes para sus textos (2013: 460). Estos autores concluyen que Twitter ofrece a los periodistas la oportunidad de acceder a la esfera privada de los protagonistas. En su estudio, más de dos tercios de los tuits se incorporan a las noticias para aportar experiencias personales, humanizar y aproximar las historias al lector.

A partir de sus hallazgos, Broersma y Graham (2013) defienden que la relación clásica entre periodistas y fuentes está cambiando, aunque matizan que disponer de un abanico rico de voces no significa que disminuya la influencia de las fuentes oficiales. No obstante, entienden que la versión de ciudadanos y fuentes alternativas, como activistas o expertos, llega de la mano de Twitter y amplía la diversidad en la cobertura de acontecimientos. Sobre esta cuestión, registran que una cuarta parte de los tuits introducidos en las noticias procede de usuarios involucrados. Las reglas del juego cambian en más sentidos. Por ejemplo, se democratiza el acceso a determinadas informaciones, antes reservadas a periodistas de gran reputación, y con acceso a fuentes valiosas. Actualmente, algunas de estas informaciones están disponibles en Twitter para todos. En contrapartida, se pierde exclusividad, lo que obliga al periodismo a redefinir su relevancia (Broersma y Graham, 2013: 461).

\section{El potencial de Twitter como (co)gatekeeper}

La literatura sobre Twitter describe también su potencial para influir en la agenda temática de los medios de comunicación. Esta capacidad le confiere el rol de (co)gatekeeper en las labores de selección y jerarquización de los asuntos noticiosos. Podría decirse que Twitter se suma al gatekeeping, proceso a través del que los periodistas filtran grandes cantidades de información para destilar un conjunto limitado de noticias en un día determinado. Su influencia sobre la agenda mediática se aprecia en diferentes sentidos:

- La red social se usa como criterio de noticiabilidad. Del mismo modo que se valora la novedad, el conflicto o el interés del protagonista antes de incluir o excluir un tema de la agenda, también se revisa si genera debate en Twitter. Si la cuestión es trending topic o tendencia, es probable que los medios se hagan eco. 
- Los debates abiertos en Twitter también se integran en las noticias. La red influye en el newsmaking o proceso de construcción de las informaciones. Lo que Twitter piensa es noticia, principalmente si los hechos generan controversia.

- Los usuarios también condicionan la agenda mediática cuando abandonan los cauces habituales de comunicación con los medios y los sustituyen por declaraciones en Twitter. Los medios pueden verse abocados a publicar aquello que interesa a las fuentes, sin posibilidad de realizar preguntas.

- Como forma de periodismo ambiental, Twitter influye en el tratamiento de los temas. Esta red social es fuente de datos, pero también de tendencias, enfoques diversos, contextos e interpretación.

- Como fuente de fuentes, Twitter pone a los periodistas en contacto con multitud de usuarios, y eso permite a los informadores encontrar pistas para nuevas coberturas o enfoques.

- Twitter es noticia. Todas las polémicas desatadas en Twitter se cuelan en la agenda mediática, en especial si están relacionadas con celebridades y personajes públicos.

Algunos autores defienden que el tradicional proceso de gatekeeping ha evolucionado en Twitter hacia el llamado gatewatching (Bruns, 2005), que incorpora a la audiencia en la tarea de evaluar el interés de los asuntos (Hermida et al., 2014). Resulta llamativo que el periodista Andy Carvin se refiera a su red de contactos en Twitter como «mis editores, investigadores y verificadores» y «mi sala de redacción» (Carvin, 2012). Por eso, Hermida et al. (2014: 495) defienden la aparición de un nuevo estilo de gatekeeping en tiempo real, porque los periodistas disponen y mencionan a un conjunto potencialmente más amplio de fuentes a través de los medios sociales. Las investigaciones sobre el movimiento $15 \mathrm{M}$ apuntan en esta misma dirección, pues demuestran que el sentir ciudadano, latente en redes sociales como Twitter, acaba imponiéndose a la agenda de medios y políticos (Casero-Ripollés y Feenstra, 2012; Castells, 2012; Micó y Casero-Ripollés, 2014; Feenstra y Casero-Ripollés, 2014).

Autores como Shoemaker y Vos (2009: 125) sugieren revisar la función del gatekeeper o periodista-guardián, en el sentido de «dejar de dar primacía» a la información generada y seleccionada por los periodistas y reconocer el impacto creciente de la información derivada de los usuarios. Las prácticas del periodista Andy Carvin durante la cobertura de las revueltas árabes se enmarcan en este contexto, pues apuntan a una remodelación de las normas clásicas y de la propia definición de periodismo, con mayor protagonismo de los usuarios en áreas hasta ahora custodiadas por los profesionales (Hermida et al., 2014: 495).

Frente a los que sostienen que la función del gatekeepig ha perdido importancia, usurpada por informadores no cualificados, autores como Bakker (2014) argumentan que los periodistas son ahora más guardianes que nunca, porque el trabajo creativo y de investigación se ha sustituido por un trabajo de oficina y gestión de contenidos. Los periodistas permanecen «encadenados a su escritorio y pegados a la pantalla, tanto para la búsqueda y curaduría de contenidos, como para solicitar, moderar y editar contenidos» (2014: 8). Tras estudiar las ofertas de trabajo para periodistas en los Países Bajos, Bakker concluye que la producción de contenido original ya no es el negocio clave. Al periodista se le pide que asuma otros roles relacionados con la gestión de contenidos. Estos nuevos roles, según Bakker, no suponen un desprestigio, sino una «actualización» del concepto de periodismo. Como ejemplo, explica que las nuevas prácticas han fomentado la especialización en el periodismo de datos, la publicación en medios sociales y la moderación de discusiones (2014: 8).

Transparencia en la redacción

Como se ha comentado, la literatura también muestra indicios de que Twitter fomenta una mayor transparencia en el proceso de elaboración de las noticias, porque algunos periodistas hablan sobre su trabajo, especialmente durante la cobertura de sucesos inesperados y revueltas (Lasorsa, 2012; Lasorsa et al., 2012; Papacharissi y De Fátima Oliveira, 2012; Artwick 2013; Hedman y Djerf-Pierre 2013; Noguera, 2013; Vis, 2013; Hermida et al., 2014). Frente al periodismo clásico, que se desarrolla en privado, los periodistas que se erigen en líderes de opinión en Twitter permiten observar al público cómo desarrollan su trabajo. Contactan con los usuarios para verificar datos y explican qué están haciendo o hacia dónde se desplazan en cada momento (Vis, 2013: 42). Sin embargo, aunque las características de Twitter permiten la gestión de los temas en público, todavía es una minoría la que ofrece una visión del funcionamiento de la redacción (Hermida, 2013).

Verificación en construcción

Diferentes estudios exponen que se descuida la tarea de verificación en Twitter (Hermida, 2013), al menos en la prensa tabloide (Broersma y Graham, 2013). Por eso, se sostiene que todos los usuarios no deberían gozar de la misma credibilidad (Hermida, 2012). Del mismo modo que para un medio convencional resulta más fiable la fuente identificada que la velada, también debería someterse a los usuariosfuente de Twitter a un proceso de autentificación. Igualmente, para sortear rumores, debería verificarse la información en circulación antes de difundirse en los medios tradicionales, a los que se presupone el cumplimiento de unas garantías mínimas (Hermida, 2010 y 2012 ; Schifferes et al., 2014).

Los usuarios de Twitter son fuente y audiencia simultáneamente, aunque conforman un público muy fragmentado, con roles muy diversos, pues entre followers y followees se incluyen amigos, familiares, fans, votantes, ciudadanos sin cualificación y profesionales en todos los campos. Algunos investigadores señalan que los políticos y famosos aprovechan Twitter para esquivar la mediación de los periodistas y colar sus consignas. Por eso, advierten que las fuentes élite pueden conseguir mayor control sobre el discurso público gracias a Twitter (Broersma y Graham, 2013: 461-462). Al respecto, apuntan la necesidad de «reporteros-curadores» para la verificación (Bruno, 2011: 67), porque los periodistas «ya no son las únicas voces que controlan el flujo informativo en la esfera pública» (Deuze, 2008: 12), pero son profesionales especializados, que pueden filtrar, sintetizar y verificar eventos en tiempo real (Newman, 2010), mientras se construye la noticia, en línea con la noción de «periodismo como proceso», no como producto acabado (Hermida, 2012: 666). 


\section{Conclusiones: limitaciones y tendencias futuras en la investigación sobre Twitter y periodismo}

Los estudios realizados hasta ahora, como se ha podido comprobar, abarcan numerosos aspectos y vertientes del impacto de Twitter en el periodismo. Sin embargo, presentan una limitación destacable. No reflejan la perspectiva de los periodistas ajenos a Twitter (Hedman y Djerf-Pierre, 2013; Hermida, 2013). Por lo tanto, pese a que las investigaciones sobre las rutinas en la redacción concluyen que el uso de Twitter se ha normalizado, debe matizarse que parten de la observación de periodistas adeptos a Twitter, y que no cuantifican ni recogen la visión de los profesionales que se mantienen al margen. A modo de ejemplo, como se ha comentado anteriormente, el $85 \%$ de los periodistas suecos afirma usar Twitter, pero sólo uno de cada diez tuitea diariamente. En consecuencia, la imagen del periodista continuamente conectado y tuiteando quedaría lejos de reflejar la realidad de la mayor parte de los profesionales (Hedman y Djerf-Pierre, 2013). Resultaría conveniente profundizar en este aspecto en futuros análisis.

En la misma línea, se percibe que predominan las investigaciones que se aproximan a Twitter desde una óptica optimista, incluso ciberutópica. Por eso, se tiende a resaltar las virtudes de Twitter y minimizar sus aspectos negativos. En este sentido, sería conveniente introducir con más énfasis investigaciones que adopten una postura crítica respecto a la incidencia de Twitter en el periodismo, para contar con otras visiones de este importante fenómeno.

Aunque el estudio de los nexos entre Twitter y el periodismo ha crecido exponencialmente en los últimos años, todavía quedan temas pendientes que pueden configurar una agenda de investigación sobre este tópico. Conformarían un catálogo de tendencias para el desarrollo de este campo en los próximos años. La primera guarda relación con la perspectiva del periodismo ambiental. Bajo este prisma, las noticias son fruto de la negociación pública y en tiempo real entre periodistas y usuarios que ejercen de fuentes. Esta reformulación del concepto de periodismo debería ser objeto de futuras investigaciones. Se aprecia en la cobertura de revueltas y sucesos imprevistos (Brown-Smith, 2012; Hermida et al., 2014), pero debería comprobarse en otro tipo de coberturas.

En relación con el papel de los usuarios en la co-construcción de las noticias, sería oportuno seguir indagando sobre la validez de Twitter como fuente periodística, con varios propósitos. Por un lado, se observa interesante establecer criterios que ayuden al periodista a distinguir a los usuarios cualificados de los tóxicos, con procedimientos para la autentificación de cuentas. Por otro lado, académicos y periodistas podrían colaborar en el diseño de protocolos comunes para la verificación de la información y las imágenes que circulan en Twitter. El estudio de casos y las entrevistas en profundidad a profesionales pueden aportar luz en estos ámbitos.

El abandono o mutación de las normas y principios clásicos del periodismo es otra vía para futuras investigaciones. Existen evidencias de que los periodistas pierden la objetividad y opinan en Twitter, y de que difunden la versión de las fuentes críticas con el poder durante la cobertura de revueltas sociales. Resultaría acertado seguir estudiando estas tendencias, para comprobar si se consolidan y en qué supuestos se practican, y analizar cómo influyen en los contenidos ofrecidos por los medios tradicionales.

Otro punto de análisis es el impacto de Twitter sobre el paradigma de la mediatización de la comunicación política. Hasta ahora, los políticos se adaptaban a la lógica de los medios para llegar a los ciudadanos. Con los nuevos medios sociales, se producen cambios importantes. Los políticos pueden dirigirse a la audiencia sin intermediarios, sorteando a los periodistas. En contrapartida, también aumenta el potencial de los usuarios para fiscalizar al poder. Por lo tanto, es apropiado analizar con más detalle si los periodistas están perdiendo capacidad de control sobre los políticos, y ahondar en cómo y en qué circunstancias los políticos cambian sus estrategias por la presión ciudadana ejercida a través de Twitter.

Los roles que el periodista ha de asumir ante los cambios descritos conforman otra línea de investigación. Parece necesaria una reflexión profunda por parte de la profesión, acerca de hacia dónde queremos ir y si es necesario retroceder en algún ámbito. El público también debería participar en el proceso de reconceptualización del periodismo, expresando su opinión a través de encuestas. En tanto que el periodismo contribuye a la formación de la opinión pública, es pertinente entender cómo valoran los ciudadanos las transformaciones que imprime Twitter en la profesión. La conculcación de normas clásicas, por ejemplo, puede estimarse positiva si se traduce en más vigilancia y control sobre los políticos.

\section{Bibliografía}

Ahmad, A. (2010). Is Twitter a Useful Tool for Journalists?. Journal of Media Practice, 11(2), 145-55.

Almaskati, N. (2012). Newspaper Coverage of the 2011 Protests in Egypt. International Communication Gazette, 74(4), 342-366.

Artwick, C. (2013). Reporters on Twitter. Digital Journalism. doi:10.1080/ 21670811.2012.744555.

Bakker, P. (2014). Mr. Gates Returns. Journalism Studies. doi:10.1080/1461670X.2014.901783.

Broersma, M., y Graham, T. (2012). Social Media as Beat. Journalism Practice, 6(3), 403-419.

Broersma, M., y Graham, T. (2013). Twitter as a News Source. Journalism Practice, 7(4), 446-464.

Brown Smith, C. (2012). \#Memstorm: Twitter as a Community-driven Breaking News Reporting Tool. \#ISOJ: The Official Journal of the International Symposium on Online Journalism, 2(2), 1-28.

Bruno, N. (2011). Tweet First, Verify Later: how real-time information is changing the coverage of worldwide crisis events. Oxford: Reuters Institute for the Study of Journalism. http://nicolabruno.files.wordpress/2011/05/tweet_first_verify_later2.pdf.

Bruns, A. (2005). Gatewatching: collaborative online news production. Nueva York: Peter Lang.

Bruns, A., y Burguess, J. (2012). Researching news discussion on Twitter. Journalism Studies, 13(5-6), 801-814. doi: $10.1080 / 1461670 X .2012 .664428$.

Burns, A. (2010). Oblique Strategies for Ambient Journalism. M/C Journal, 13(2). http://journal.mediaculture.org.au/index.php/mcjournal/article/view/230 (28/08/2012).

Carrera Álvarez, P., Sainz de Baranda, C., Hererro, E., y Limón, N. (2012). Journalism and Social Media: How Spanish Journalists Are Using Twitter. Estudios sobre el mensaje periodístico, 1(18), 31-53.

Carvin, A. (2012). I don't just have Twitter followers. You're my editors, researchers and act-checkers. You're my news room. And I dedicate this award to you. Post en Twitter, 26 de marzo. http://twitter.com/acarvin/status/184424440757624832. 
Casero-Ripollés, A., y Feenstra, R. (2012). The 15-M Movement and the new media: A case study of how new themes were introduced into Spanish political discourse. Media International Australian, 144, 68-76.

Castells, M. (2009). Comunicación y poder. Madrid: Alianza Editorial.

Castells, M. (2012). Networks of outrage and hope: Social movements in the internet age. Cambridge: Polity.

Cozma, R., y Chen, K. (2013). What's in a Tweet?. Journalism Practice, 7(1), 33-46. doi: 10.1080/17512786.2012.683340.

Deuze, M. (2008). Understanding Journalism as Newswork: how it changes, and how it remains the same. Westminster Papers in Communication and Culture, 5(2), 4-23.

Domingo, D., Quandt, T., Heinonen, A., Paulussen, S., Singer, J. B., y Vujnovic, M. (2008). Participatory Journalism Practices in the Media and Beyond. Journalism Practice, 2(3), 326-342. doi: 10.1080/17512780802281065.

Ekman, M., y Widholm, A. (2014). Politicians as Media Producers. Journalism Practice. doi: 10.1080/17512786.2014.928467.

Farhi, P. (2009). The Twitter Explosion. American Journalism Review, 31(3), 26-31.

Feenstra, R., y Casero-Ripollés, A. (2014). Democracy in the Digital Communication Environment: A Typology Proposal of Political Monitoring Processes. International Journal of Communication, 8, 2448-2468.

Ferguson, D. A., y Greer, C. F. (2011). Local Radio and Microblogging: how radio stations in the U.S. are using Twitter. Journal of Radio and Audio Media, 18(1), 33-46.

Flores, J. (2009). Nuevos modelos de comunicación, perfiles y tendencias en las redes sociales. Comunicar, 33, 73-81.

García Avilés, J. A., Kaltenbrunner, A., y Meier, K. (2014). Media Convergence Revisited. Journalism Practice. doi:10.1080/17512786.2014.885678.

García de Torres, E., Yezers'ka, L., Rost, A., Calderin, M., Rojano, M., Edo, C., ... \& Corredoira, L. (2011). See you on Facebook or Twitter? The use of social media by 27 news outlets from 9 regions in Argentina, Colombia, Mexico, Peru, Portugal, Spain and Venezuela. En 12th International Symposium for Online Journalism, Austin. http://online.journalism.utexas.edu/2011/papers/Elvira2011.pdf.

Greer, C. F., y Ferguson, D. A. (2011). Using Twitter for Promotion and Branding: A Content Analysis of Local Television Twitter Sites. Journal of Broadcasting and Electronic Media, 55(2), 198-214. doi: 10.1080/08838151.2011.57082.

Greer, J. D., y Yan, Y. (2010). New Ways of Connecting with Readers. How community newspapers are using Facebook, Twitter and other social tools to deliver the news. Grassroots Editor, 51(4), 1-7.

Gulyas, A. (2013). The Influence of Professional Variables on Journalists' Uses and Views of Social Media. A Comparative Study of Finland, Germany, Sweden and the United Kingdom. Digital Journalism. doi:10.1080/21670811.2012.744559.

Hedman, U., y Djerf-Pierre, M. (2013). The Social Journalist: Embracing the Social Media Life or Creating a New Digital Divide?. Digital Journalism.

doi:10.1080/ 21670811.2013 .776804 .

Hermida, A. (2010). Twittering the news: The emergence of ambient journalism. Journalism Practice, 4(3), 297-308. doi: 10.1080/17512781003640703.

Hermida, A. (2012). Tweets and truth: Journalism as a Discipline of Collaborative Verification. Journalism Practice, 6(5-6), 659-668. doi: 10.1080/17512786.2012.667269.

Hermida, A. (2013). \#Journalism. Digital Journalism, 1(3), 295-313. doi: 10.1080/21670811.2013.808456.

Hermida, A., Domingo, D., Heinonen, A., Paulussen, S., Quandt, T., Reich, Z., ... y Vujnovic, M. (2011). The active recipient: Participatory journalism through the lens of the Dewey-Lippmann debate. En International Symposium on Online Journalism, 1(2). http://online.journalism.utexas.edu/2011/papers/Hermida2011.pdf.

Hermida, A., Lewis, S. C., y Zamith, R. (2014). Sourcing the Arab Spring: A Case Study of Andy Carvin's Sources on Twitter during the Tunisian and Egyptian Revolutions. Journal of Computer-Mediated Communication, 19, 479-499. doi: 10.1111/jcc4.12074.

Hermida, A., y Thurman, N. (2008). A Clash of Cultures: the Integration of User-generated Content within Professional Journalistic Frameworks at British Newspaper Websites. Journalism Practice, 2, 343-356. doi: 10.1080/17512780802054538.

Herrera, S., y Requejo, J. L. (2012). 10 Good Practices for News Organizations Using Twitter. Journal of Applied Journalism and Media Studies, 1(1), 79-95. doi: 10.1386/ajms.1.1.79_1.

Holcomb, J., Gross, K., y Mitchell, A. (2011). How Mainstream Media Outlets Use Twitter: Content Analysis Shows Evolving Relationship. Project for Excellence in Journalism. http://www.journalism.org/node/27311 (16/07/2014).

Java, A., Song, X., Fnin, T., y Tseng, B. (2007). Why We Twitter: Understanding Microblogging Usage and Communities. En Proceedings of the 9th WebKDD and 1st SNA-KDD 2007 Workshop on Web Mining and Social Network Analysis. Nueva York: ACM Press, 56-65. doi: 10.1145/1348549.1348556.

Lasorsa, D. (2012). Transparency and Other Journalistic Norms on Twitter. Journalism Studies, 13(3), 402-417. doi: 10.1080/1461670X.2012.657909.

Lasorsa, D., Lewis, S. C., y Holton, A. (2012). Normalizing Twitter: Journalism Practice in an Emerging Communication Space. Journalism Studies, 13(1), 19-36. doi: 10.1080/1461670X.2011.571825.

López, X., y Alonso, S. (2013). Los periódicos gallegos en las redes sociales virtuales: presencia y posicionamiento en el nuevo escenario comunicativo. Estudios sobre el Mensaje Periodístico, 19(2), 1001-1016.

Lotan, G., Graeff, E., Annanny, M., Gaffney, D., Pearce, I., y Boyd, D. (2011). The Revolutions Were Tweeted: Information Flows during the 2011 Tunisian and Egyptian Revolutions. International Journal of Communication, 5, 1375-1405. http://ijoc.org/ojs/index.php/ijoc/article/view/1246 (16/07/2014).

Messner, M., Linke, M., y Eford, A. (2012). Shoveling Tweets: An Analysis of the Microblogging Engagement of Traditional News Organizations. \#ISOJ: The Official Research Journal of the International Symposium on Online Journalism, 2(1), 76-90.

Micó, J. LL., y Casero-Ripollés, A. (2014). Political activism online: organization and media relations in the case of 15M in Spain. Information, Communication and Society, 17(7), 858-871.

Newman, N. (2009). The Rise of Social Media and Its Impact on Mainstream Journalism. Oxford: Reuters Institute for the Study of Journalism.

http://reutersinstitute.politics.ox.ac.uk/fileadmin/documents/Publications/The rise of social_media_and_its_impact_on_mainstream_journalism.pdf (24/08/2012).

Newman, N. (2010). Journalism and Technology Predictions 2011. The Media Briefing. http://www.themediabriefing.com/resource/mediamarket-journalism-and-technology-predictions-2011-by-nic-newman (24/08/2012).

Noguera, J. M. (2013). How Open Are Journalists on Twitter? Trends Towards the End-user Journalism. Communication and Society, 26(1), 93-114. 
Papacharissi, Z., y De Fátima Oliveira, M. (2012). Affective News and Networked Publics: The Rhythms of News Storytelling on \#Egypt. Journal of Communication, 62, 266-282. doi: 10.1111/j.1460-2466.2012.01630.x.

Poell, T., y Borra, E. (2011). Twitter, YouTube, and Flickr as platforms of alternative journalism: The social media account of the 2010 Toronto G20 protests. Journalism. doi:10.1177/1464884911431533.

Requejo, J. L., y Herrera, S. (2014). Retroceso en la creación de comunidad entre los diarios generalistas españoles en Twitter (2011-2013). Estudios sobre el Mensaje Periodístico, 20(1), 229-248.

Rodríguez, A., y García, J. (2013). Uso y funciones de Twitter en periodistas españoles. Estudios sobre el mensaje periodístico, 19, especial abril, 963-969.

Said, E., Serrano, A., García de Torres, E., Yerezers'ka, L., y Calderín, M. (2013). La gestión de los Social Media en los medios informativos iberoamericanos. Comunicación y Sociedad, 26 (1), 67-92.

Sakaki, T., Okazaki, M., y Matsuo, Y. (2010). Earthquake shakes Twitter users: Real-time event detection by social sensors. En Proceedings of the 19th international conference on World Wide Web, WWW '10. New York: ACM Press, 851-860. doi:10.1145/1772690.1772777.

Salaverría, R., García Avilés, J. A., y Masip, P. (2010). Concepto de convergencia periodística. En López, X., y Pereira, X. (coords.). Convergencia Digital. Reconfiguración de los Medios de Comunicación en España, 41-64. Santiago de Compostela: Universidad de Santiago de Compostela.

Schifferes, S., Newman, N., Thurman, N., Corney, D., Göker, A., y Martín, C. (2014). Identifying and Verifying News through Social Media. Digital Journalism. doi: 10.1080/21670811.2014.892747.

Sheffer, M. L., y Schultz, B. (2010). Paradigm Shift or Passing Fad? Twitter and Sports Journalism. International Journal of Sport Communication, 3, 472-484.

Shoemaker, P. J., y Vos, T. P. (2009). Gatekeeping Theory. New York: Routledge.

Singer, J., Hermida, A., Domingo, D., Heinonen, A., Paulussen, S., Quandt, T., ...Vujnovic, M. (2011). Participatory Journalism: Guarding Open Gates at Online Newspapers. New York: Wiley-Blackwell.

Starbird, K., Muzny, G., y Palen, L. (2012). Learning from the Crowd: Collaborative Filtering Techniques for Identifying On-the-ground Twitters during Mass Disruptions. En Proceedings of the Conference on Information Systems for Crisis Response and Management (ISCRAM 2012). Vancouver: Simon Fraser University.

Túñez, M. (2012). Los periódicos en las redes sociales: audiencias, contenido, interactividad y estrategias comerciales. Estudios sobre el mensaje periodístico, 18(1), 221-239.

Vis, F. (2013). Twitter As a Reporting Tool for Breaking News. Digital Journalism, 1(1), 27-47. doi: 10.1080/21670811.2012.741316.

Wickre, K. (2013). Celebrating \#Twitter. Twitter Blog, 21 de marzo. http://blog.twitter.com/2013/03/celebrating-twitter7.html (20/10/2014).

\section{Cómo citar este artículo en bibliografías - How to cite this article in bibliographies / references:}

LÓPEZ-MERI, A. (2015): "El impacto de Twitter en el periodismo: un estado de la cuestión". En Revista de la Asociación Española de Investigación de la Comunicación, vol. 2, número 4, pp. 34-41. 